\title{
Fabrication of Frequency-Selective Surface Structures by Femtosecond Laser Ablation of Gold Films
}

\author{
Vygantas Mizeikis, $^{*}$ Saulius Juodkazis ${ }^{* * * * * *}$, Kai Sun ${ }^{* * *}$, and Hiroaki Misawa ${ }^{* *}$ \\ * Division of Global Research Leaders and Research Institute of Electronics , Shizuoka University, \\ 3-5-1 Johoku, Naka-ku, Hamamatsu 432-8561, Japan \\ dvmzks@ipc.shizuoka.ac.jp \\ ${ }^{* *}$ Research Institute for Electronic Science (RIES), Hokkaido University, N21 W10 CRIS Bldg., \\ Sapporo 001-0021, Japan
}

\begin{abstract}
${ }^{* * *}$ Hokkaido Innovation Through Nanotechnology Support (HINTS) and Research Institute for Electronic Science (RIES), Hokkaido University, N21 W10 CRIS Bldg., Sapporo 001-0021, Japan

\author{
${ }^{* * *}$ Centre for Micro-Photonics, Faculty of Engineering and Industrial Sciences, Swinburne \\ University of Technology, Hawthorn, VIC, 3122, Australia
}

\begin{abstract}
Simple frequency-selective surface (FSS) structures consisting of two-dimensional (2D) triangular lattices of circular apertures with an aperture diameter of about $0.6-0.8 \mu \mathrm{m}$ and a lattice period of 1.0 and $2.0 \mu \mathrm{m}$ were fabricated by femtosecond laser ablation of thin gold films deposited on glass substrates. The fabricated samples were found to exhibit spectral transmission bands at infrared wavelengths, and significant surface plasmonic (SP) local enhancement of the near-field near the metal's surface according to experimental transmission measurements and theoretical simulation by Finite-Difference Time-Domain (FDTD) technique. These results indicate versatility of femtosecond laser ablation technique for rapid prototyping of metallic FSS and plasmonic structures.
\end{abstract}

DOI:10.2961/jlmn.2010.02.0003

Keywords: Frequency-selective surfaces, femtosecond laser ablation

\section{Introduction}

Frequency-selective surfaces (FSS)[1] are periodic dielectric or metallic planar structures which can perform spectral, spatial, or angular filtering of optical radiation owing to shape and size of the constituent features, as well their periodic arrangement. Metallic FSS structures consisting of periodic arrays of sub-wavelength apertures or patches are widely used for controlling flow of electromagnetic radiation in the microwave and millimeter spectral regions [2-4]. More recently, a wider class of artificially structured electromagnetic metamaterials [5] with characteristics not found in natural materials (e.g., negative refraction index) has emerged, which shares many similarities with FSS. Active wavelength range in which these structures exhibit characteristic properties is typically close to their feature size and spatial period. While FSS structures for longer wavelengths can be fabricated by mechanical machining, tuning their active wavelength to shorter, infrared (IR) and near-infrared (NIR) spectral ranges requires higher resolution of fabrication. Micro-and nano-structuring of thin films of low-loss noble metals (Such as $\mathrm{Au}, \mathrm{Ag}, \mathrm{Al}$, and $\mathrm{Cu}$ ) deposited on dielectric or semiconducting substrates typically involves lithographic definition of pattern, deposition of metallic film, pattern lift-off, or ion-beam milling of the deposited films. Although such approach has proven capable of delivering high-quality micro- and nanostructured metallic surface structures on dielectric substrates [6], one would often benefit from a fast, singlestep cost-efficient fabrication methods [7]. Here, we report use of femtosecond laser ablation of thin gold films deposited on transparent glass substrates to produce simple prototypes of FSS structures for IR spectral range. Previously, femtosecond laser ablation of metallic surfaces in liquids was successfully used for fabrication of metallic nanoparticles capable of supporting surface plasmons [8]. However, these methods allowed fabrication of nanoparticles suspended in a liquid and having random size, shape, and orientation. Our approach to fabrication of FSS surfaces is illustrated schematically in Fig. 1(a). Single femtosecond laser pulses are tightly focused on a thin metal film (in our case, gold) which becomes locally removed by ablation leaving circular apertures in the film. Periodic scanning of the focal spot synchronously with arrival of the laser pulses allows one fabricate ordered arrays of apertures in the gold film. Such fabrication is maskless and essentially a single-step process. In comparison to metal ablation in liquids [8], this technique allows fabrication of 



Fig. 1. (a) The principle of FSS structure fabrication by ablation of a thin gold film, (b) triangular structure of laser-ablated apertures and its geometrical parameters

somewhat better ordered structures attached to solid substrate.

For this study, triangular lattice of apertures, which is shown schematically in Fig. 1(b) together with tentative lattice parameters (to be discussed later) were chosen and successfully fabricated by the method outlined above. The resulting FSS structures were found to have good structural quality, and exhibit spectral bands of enhanced transmission at infrared wavelengths in the range of 1.5$3.0 \mu \mathrm{m}$, according to experimental measurements of their transmission spectra and theoretical modeling using FiniteDifference Time-Domain (FDTD) technique. The simulations also indicate that within the transmission bands, significant enhancement of the near-field intensity occurs close to the edges of apertures in the gold film, indicating importance of surface plasmons (SP) for the enhanced transmission. These results indicate the versatility of the proposed fabrication method for fast, cost-efficient prototyping of metallic FSS structures for IR spectral range.

\section{Fabrication procedure and samples}

Optical setup for fabrication is shown schematically in Fig. 2. The laser source is a femtosecond Hurricane $X$ system (Spectra-Physics) with a pulse duration $\tau_{\mathrm{p}}=130 \mathrm{fs}$, a central wavelength of $\lambda_{p}=800 \mathrm{~nm}$, and a repetition rate adjustable within the $1 \mathrm{~Hz}-1 \mathrm{kHz}$ range. The laser beam has a Gaussian profile with a full width at half maximum (FWHM) of $4 \sim \mathrm{mm}$. The beam is attenuated by a variable attenuator, and its divergence is compensated by an optical telescope (not shown) prior to coupling into an inverted optical microscope (Olympus IX71) which houses focusing objective lenses and a sample holder. An oil-immersion lens having a numerical aperture (NA) of 1.4 and an air lens with NA=0.9 were used for focusing of the laser beam on the gold film deposited on microscope cover-glass substrates. The samples were mounted on a high-precision

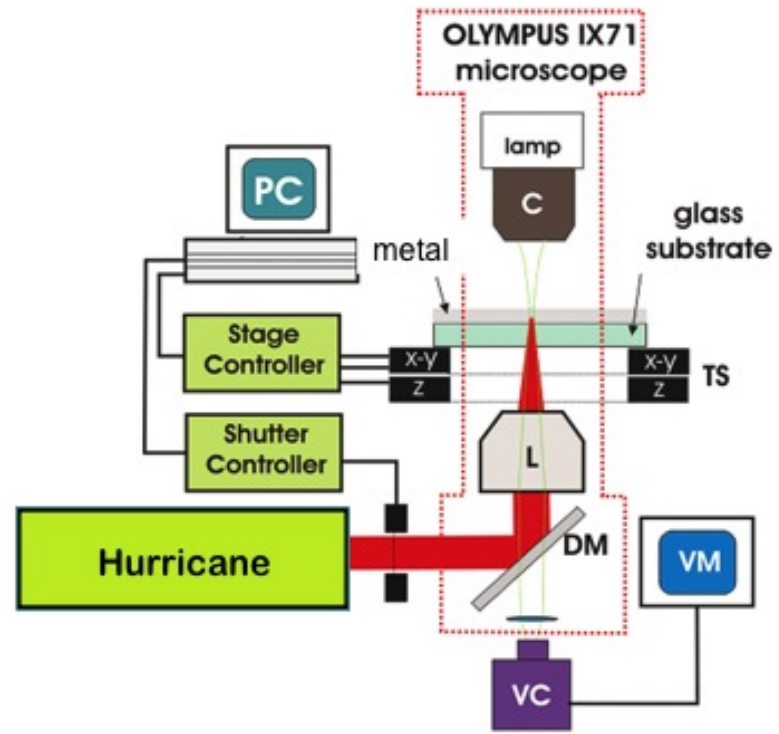

Fig. 2. Experimental setup for optical fabrication, abbreviations: microscope lens (L), dichroic mirror (DM), three-dimensional translation stage (TS), video camera (VC), video monitor (VM), condenser (C), personal computer (PC).

piezoelectric transducer (PZT) controlled 3D translation stage consisting of paired $x-y$ and $z$ stages (Physik Instrumente P-517.2CL, and P-518.ZCL, respectively), attached to the microscope. This setup provides positioning accuracy of several nanometers within the maximum range of $(100 \times 100 \times 100) \mu^{3}$. Although the task of planar patterning requires repositioning of the sample in $x-y$ plane only, adjustment of its $z$-axis coordinate is helpful in optimizing the focusing depth to the surface plane of the gold film. Prior to the fabrication, tilt of the substrate was carefully adjusted in order to achieve identical focusing conditions across the entire area to be perforated. During the fabrication the samples were monitored in-situ under illumination of a halogen lamp using a video camera. Fabrication was performed at a relatively slow laser repetition rate of $15 \mathrm{~Hz}$ in order to provide time intervals sufficient for the translation stage to move between the neighboring ablation spots, and an additional waiting time of a few milliseconds prior to arrival of the laser pulse for mechanical relaxation of the stage. Fabrication procedure as well as extent and spatial arrangement of the ablation areas were controlled by a personal computer. Two sets of samples with lattice period $a=2.0 \mu \mathrm{m}$ and $1.0 \mu \mathrm{m}$ were prepared for these studies as indicated in Fig. 1(b). All structures reported below were fabricated in a single-shot irradiation regime. Diameter of the laser-ablated apertures was controlled by adjusting the laser pulse energy. Typically, single pulse energies of about 5 and $15 \mathrm{~nJ}$ were required using $\mathrm{NA}=1.4$ and $\mathrm{NA}=0.9$ objective lenses, respectively, in order to produce circular apertures with diameter approaching the laser wavelength. Fig. 1(b) gives the range of aperture diameters of $d=0.6-1.0 \mu \mathrm{m}$, which represents the approximate practically achievable range under our experimental conditions (see Fig. 3 below). 

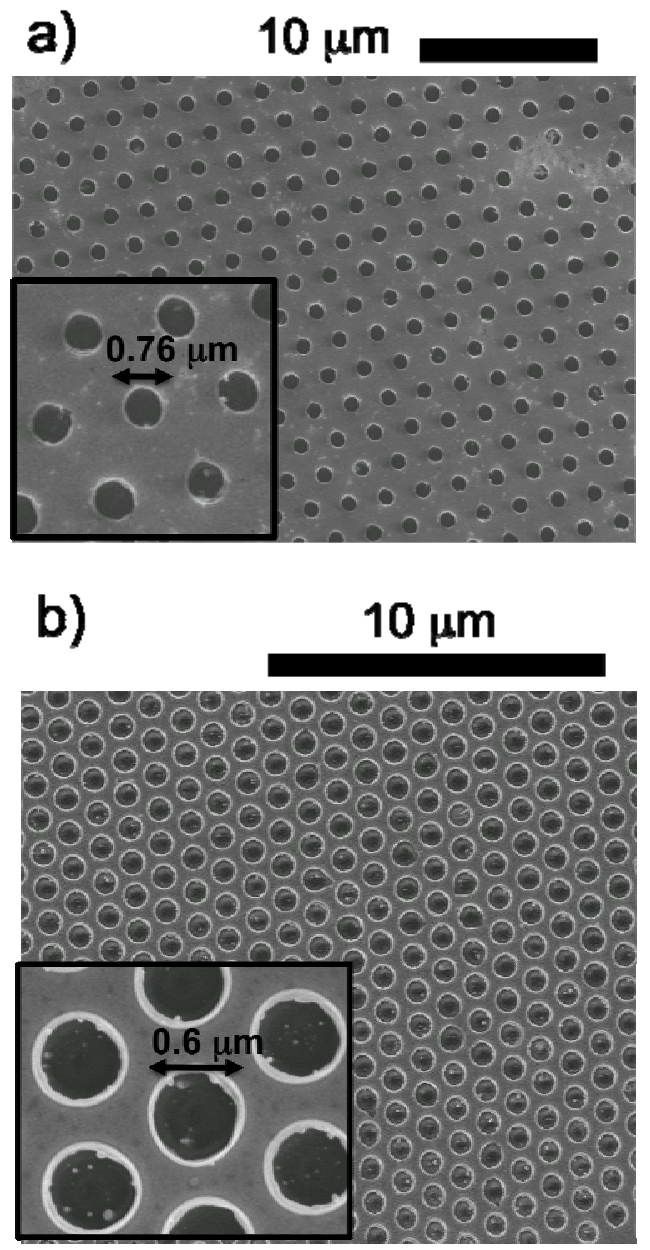

Fig. 3. SEM images of FSS samples, (a) structure parameters $\mathrm{a}=2.0 \mu \mathrm{m}, \mathrm{d}=0.76 \mu \mathrm{m}$, focusing by $\mathrm{NA}=1.4$ oil-immersion lens, laser pulse energy $5.0 \mathrm{~nJ}$, (b) structure parameters $\mathrm{a}=1.0 \mu \mathrm{m}, \mathrm{d}=0.6 \mu \mathrm{m}$, focusing by $\mathrm{NA}=0.9$ air lens, laser pulse energy $15.0 \mathrm{~nJ}$.

The initial samples were gold films deposited by sputtering to a thickness $h=36 \mathrm{~nm}$, as determined using atomic-force microscopy (AFM), on microscope coverglass substrates (Matsunami) with a size of $(24 \times 24) \mathrm{mm}^{2}$, and a thickness of $0.17 \mathrm{~mm}$. It is worth noting that fabrication of gold nanostructures on glass substrates by other techniques (such as lithography and lift-off) would require a thin primer layer of $\mathrm{Cr}$ or Ti between the substrate and gold film in order to improve adhesion and withstand lift-off. However, no primer layer was necessary in the case of laser ablation, which essentially combines mask-less pattern definition and lift-off steps.

As noted above, optical fabrication of various samples was performed using two different objective lenses for the focusing: the oil-immersion lens with NA=1.4 and the air lens with $\mathrm{NA}=0.9$. The first of these lenses produces extremely tight focusing and allows one obtain focal spot size comparable to the laser wavelength. The use of immersion oil restricts one to so-called back-side ablation geometry shown in Fig. 1(a), in which laser beam propagates across the substrate before hitting its "back side" coated by the gold film. The second microscope lens used is less tightly focusing, but since it does not use immersion liquid, it enables both back-side and direct front-side ablation (i.e., with beam incidence from the side of gold film). In our preliminary experiments, both backside and front-side ablation were attempted, but the former was generally more efficient, and produced better-shaped apertures with fewer byproducts (such as gold melting and random debrii). The advantage of back-side ablation is most likely due to the circumstance that laser pulse is incident directly on the interface between the substrate and the gold film thus helping to efficiently dislodge it. Below, we consider only the samples obtained in the back-side ablation regime.

\section{Results and discussion}

Structural properties and quality of the samples were examined using scanning electron microscopy (SEM). Figure 3 shows images of two samples with different lattice parameters and fabricated under different conditions. The first sample shown in Fig. 3(a) has lattice period $a=2.0 \mu \mathrm{m}$ and aperture diameter $\mathrm{d}=0.76 \mu \mathrm{m}$, and was fabricated under focusing by NA=1.4 oil-immersion lens with laser single pulse energy of $5.0 \mathrm{~nJ}$ at the focus. The total area of the structure was $(80 \times 80) \mu \mathrm{m}^{2}$. Despite relatively low laser repetition frequency, fabrication of one structure took less than one minute. Under these circumstances control of lattice period with accuracy of few nanometers is possible. The diameter of ablation apertures is controlled by the laser pulse energy. We have optimized the pulse energy for smallest aperture diameter by recording sets of test structures with gradually decreasing laser pulse energy. We have found from these experiments that using the NA=1.4 oil immersion lens, pulse energies should not exceed 5-7 nJ in order to obtain apertures with circular shape, and diameter approaching the laser wavelength. At higher pulse intensities the apertures increased in diameter (due to significantly exceeded ablation threshold) and became noncircular (most likely due to imperfect transverse shape of the laser beam). Moreover, significant ablative damage pits with lateral size of $200-300 \mathrm{~nm}$ developed on the substrate. Reducing the pulse energy to the level of 5-7 nJ resulted in ablation of gold film only, without any damage of the substrate, as is evident from Fig. 3(a). Adhesion of gold film to glass is typically poor (usually, a thin primer layer of $\mathrm{Ti}$ or $\mathrm{Cr}$ is used to improve adhesion), and direct ablation at the Au-glass interface helps dislodge circular regions of gold film without visible damage to the substrate. On the other hand, we found it difficult to further reduce the diameter of circular ablation apertures by decreasing the pulse energy. At lower pulse energies, the ablation was partial (strongly non-circular), and its degree became somewhat random, most likely due to the pulse-to-pulse instability of the laser. Thus, under the circumstances of this work, the optimum single pulse energy is $5-7 \mathrm{~nJ}$, at which circular apertures with diameter $d=0.76 \mu \mathrm{m}$, i.e., slightly smaller than the laser wavelength, can be obtained.

Figure 3(b) shows SEM image of another FSS sample with parameters $a=1.0 \mu \mathrm{m}$ and $d=0.6 \mu \mathrm{m}$, fabricated using a less tightly focusing lens with $\mathrm{NA}=0.9$. Correspondingly, higher laser pulse energy of $15.0 \mathrm{~nJ}$ was found to be 
optimum for ablation of circular apertures. Curiously, the minimum aperture diameter obtained using this lens is slightly lower than that obtained in the case of tighterfocusing oil-immersion lens (compare Fig. 3(a) and (b)).

It is evident from images in Fig. 3 that laser ablation can produce fairly uniform arrays of circular apertures in thin gold films. Even better results can be expected by improving pulse-to-pulse stability of the laser. At the same time, formation of thicker Au rings near the edges of apertures is evident. The rings may be either a consequence of gold melting, or its partial "un-sticking" from the substrate near the aperture edges. Inspection of the fabricated structures by AFM has revealed that melting rings have maximum width of about 20-30 nm and height (with respect to the surface of unprocessed areas) of about $15 \mathrm{~nm}$. Although melting leads to structural deviations from the idealized structure shown in Fig. 1(b), it occurs at every aperture and therefore does not affect uniformity of the array significantly. Another side effect of ablation is formation of randomly dispersed spheroidal gold nanoparticles with sizes not exceeding few tens of nanometers, which can be seen in Fig. 3(b). Formation of metallic nanoparticles by laser ablation is undesirable, since gold nanoparticles are likely to cause intense elastic light scattering [9] and degrade optical uniformity of the structure. However, additional "optical cleaning" by applying subsequent laser pulses to the ablated apertures may melt these nanoparticles, or remove them from the surface, thus reducing their undesirable effect.

Optical properties of structures shown in Fig. 3 were characterized experimentally by measurements of their infrared transmission spectra, and by theoretical simulations of the transmission spectra and spatial distribution of near-field using FDTD technique [10]. The experimental spectra were measured using FourierTransform Infra-Red (FT-IR) spectrometer FT/IR-6000TM$M$ (Jasco) equipped with an in infrared microscope attachment. The use of microscope enables convenient performance of optical measurements on areas with lateral dimensions as small as few micrometers. The infrared microscope uses Cassegrain reflection objectives for the formation of probing infrared beam incident on the sample, and for collection of the transmitted signal. The construction of Cassegrain objectives essentially eliminates radiation propagating along their optical axis, limiting their angular acceptance range to a hollow cone defined by their minimum and maximum acceptance angles. The microscope used in these studies had angular acceptance range of $16^{0}-32^{0}[11]$. Since FSS structures are generally sensitive to the incidence and observation angle, this fact has to be taken into account during theoretical modeling. During the measurements, the gold film was oriented normal to the optical axis of the objectives.

FDTD Solutions software package (Lumerical, Inc.) was used for FDTD modeling. The geometry of model structures was defined using parameters of the FSS samples determined from SEM and AFM observations. The effects of melting (formation of thicker rings) were ignored in the simulations. Optical properties of gold were described using polynomial approximation of the available experimental data [12]. Glass substrate was assumed to behave as a dielectric with constant refractive index $n=$


Fig. 4. Measured (solid lines) and simulated (longdashed lines) IR transmission spectra for (a) the sample shown in Fig. 3(a), (b) the sample shown in Fig. 3(b). In (b), spectral dependence of near-field intensity enhancement factor on the surface plane of gold film calculated by FDTD technique is also shown (shortdashed line).

1.47. The simulations were performed by launching a short, spectrally broad radiation pulse as a plane wave propagating along the normal to the surface of perforated gold film, and calculating its propagation in a timestepping algorithm according to discretized set of Maxwell's equations, and by recording the spatio-temporal evolution of transmitted pulse on a plane located behind the sample in the near-field domain. The simulations were performed within one unit cell of the periodic structure with periodic boundary conditions imposed at its boundaries, while perfectly-matched boundary conditions were imposed on boundaries parallel to the gold film in order to allow reflected and transmitted radiation freely escape the calculation domain. Discrete rectangular mesh with spacing of $5 \mathrm{~nm}$ was used for the simulations. As noted above, normal incidence of the probing pulse on the gold film was assumed, thus neglecting the actual angular range of incidence angles due to the use of Cassegrainian objective. However, we have found that if simulations were carried with angularly-limited detection (see below), their results were relatively independent of the incidence angle. For the transmitted radiation, the limited angular range of collection was accounted for by performing near-to-farfield transform of the transmitted field (detected at a 
monitor plane located in the near-field), and by its subsequent angular filtering within the experimental angular range of $16^{0}-32^{0}$. It is helpful to emphasize here, that perforated gold films exhibit strong elastic light scattering in a spatial angle approaching $4 \pi$, i.e., somewhat wider than the experimental detection range. This circumstance may help understand relative insensitivity of the simulated results on the incidence angle. As will be shown below, a reasonable agreement between simulations and experiments was achieved under the assumptions used.

Measured and simulated IR transmission spectra of the samples shown in Fig. 3, are summarized in Fig. 4. Both samples exhibit transmission bands, which have different spectral shapes, but fall into the same wavelength range of 1.5-3.5 $\mu \mathrm{m}$. Generally, transmission modulation is not very pronounced in both samples, but is slightly stronger in the second sample (Fig. 4(b)) which has higher fraction of surface area occupied by the apertures (note, that transmission of unstructured gold film with thickness of $36 \mathrm{~nm}$, about 0.01 , is insignificant compared to that of patterned samples. For the first sample (Fig. 4(a)), rising edge of an additional transmission band can be seen as the wavelength decreases to $1.0 \mu \mathrm{m}$ and beyond. This increase in the signal is most likely due to angular matching between the higher diffracted orders, which become propagating waves for wavelengths shorter than the lattice period $a=2.0 \mu \mathrm{m}$. For comparison, the second sample with shorter lattice period $a=1.0 \mu \mathrm{m}$ does not exhibit enhanced transmission at shorter wavelengths.

The low modulation amplitude of experimentally measured transmission bands is most likely the result of angularly-limited detection geometry peculiar to Cassegrainian objectives. In this work we did not have an opportunity to fabricate large-area FSS samples suitable for optical probing and detection using collimated, normally incident/exiting beams, and therefore could not experimentally verify this assumption. Its theoretical verification was done using FDTD simulations of transmission spectra performed along the normal to the surface of gold film. As can be expected, these simulations have revealed stronger modulation of transmission, but on the other hand, spectral positions and shapes of the experimental transmission bands could not be reproduced. Taking into account the limited range of spatial angles for detection of transmitted signal has allowed qualitative reproduction of the measured spectra, as is illustrated by the results of FDTD simulations shown in Fig. 4(a) and (b). Therefore, we conclude that the appearance of measured transmission bands is partly caused by intrinsic transmission properties of the structures, and partly by the excitation and detection conditions used. The simulations cannot produce exact shape of the measured transmission spectra, because they ignore finer features of the sample (such as thicker Au rings near the edges of apertures), and therefore there is some mismatch between spectral positions and linewidths of the simulated and experimental transmission bands.

These observations and theoretical simulations suggest that the investigated samples fabricated by ablation of thin gold films indeed exhibit basic functionality of FSS
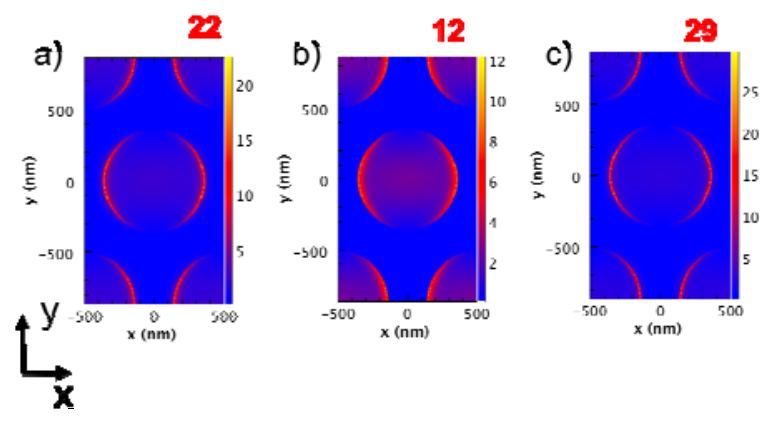

Fig. 5. Near field intensity distribution calculated by FDTD technique for the sample shown in Fig. 3(b) on horizontal planes coincident with (a) top of the gold film , (b) half-height of the gold film, and (c) interface between the gold film and the glass substrate. The field intensity was normalized to that of the incident field, and therefore represents the near-field enhancement factor. The maximum field intensity enhancement factors are indicated by numbers. The incident wave was polarized linearly parallel to $x$-axis.

structures. It is instructive to examine briefly the physical mechanism responsible for the observed transmission bands. Insight to this mechanism can be gained by examining near-field patterns obtained from FDTD simulations. Figure 5 shows near-field spatial distribution calculated for the second sample $(a=1.0 \mu \mathrm{m}, d=0.6 \mu \mathrm{m}$, $h=36 \mathrm{~nm}$, see Fig. 3(b)) on three selected planes parallel to the surface of the gold film at various heights above the glass-metal interface. The field patterns were simulated at the wavelength of $1.35 \mu \mathrm{m}$, within the FSS transmission band. They are normalized to the intensity of the incident wave, and thus essentially represent the near-field intensity enhancement factor. All three patterns in Fig. 5 indicate predominant localization of the near-field inside the apertures, where intensity enhancement by approximately 5 times is reached. Even stronger field localization occurs near the edges of apertures, where intensity enhancement factor reaches from 12 to 29 on various planes. The nearfield enhancement is spectrally dependent, and its spectral shape approximately matches that of the transmission band. This circumstance is illustrated in Fig. 4(b), where nearfield enhancement factor calculated on the top of the gold film is plotted against the wavelength. The enhancement factor has a peak near the wavelength of $1.35 \mu \mathrm{m}$, which is the wavelength of choice for the near-field patterns in Fig. 5.

Formation of resonant bands characterized by near-field localization and intensity enhancement is common to nanostructures of noble metals[9,13-15], and is widely associated with surface plasmons (SP), which are coupled oscillations of the electromagnetic wave and free electrons of the metal. SP are known to play crucial role in promoting transmission through sub-wavelength apertures and aperture arrays [16,17]. Comparison between conditions and findings of this study and reports available in the literature [16-19] suggests a strong role of SP effects 
in the observed infrared transmission bands in our FSS samples. Thus, resonant transmission bands, and their finer features seen in Fig. 4 represent various SP modes supported by the structure. Detailed investigation of SP modal patterns at various wavelengths goes beyond the scope of this work and will be published in future.

\section{Conclusions}

Using femtosecond laser ablation of thin gold films metallic FSS structures consisting of triangular lattice of circular apertures with an aperture diameter of about 0.6$0.8 \mu \mathrm{m}$ and a lattice period of 1.0 and $2.0 \mu \mathrm{m}$ were fabricated. Optical investigation of the structures by transmission measurements and theoretical FDTD simulations reveal spectral transmission bands at infrared wavelengths, which are most likely associated with formation of surface plasmons, as is evident from resonant localization of the near-field within the transmission bands and the associated enhancement of the near-field intensity near the edges of laser-ablated apertures. Besides the spectral modulation of transmission, similar structures can be interesting candidates in applications relying on the near-field enhancement, such as promotion of nonlinear optical phenomena at infrared wavelengths (for example, non-linear photoluminescence) in the nanoscale, since the estimated field intensity enhancement by the factor of approximately 20 would increase the yield of non-linear processes by the factor of approximately 400 (in the case of square intensity dependence). Coincidentally, resonant bands in some of the fabricated structures partially overlap with optical communications wavelength range (1.3$1.6 \mu \mathrm{m}$ ), which is attractive from the applications point of view. Although this study is far from being systematic, it already indicates the potential of femtosecond laser ablation for fast, single-step prototyping of FSS structures, and raises some questions that might attract further investigations. In particular, influence of structure periodicity and shapes of individual elements [16,17] (in our case, circular apertures) as well as thickness of metallic film [18] on optical properties of FSS structures should be clarified. From the viewpoint of fabrication process, possibilities to use films of other noble metals (Ag, $\mathrm{Al}, \mathrm{Cu}$ ), and to increase spatial resolution achievable by ablation would certainly expand versatility of this method and allow fabrication of FSS structures with more complex topologies for shorter active wavelengths.

\section{References}

[1] B. A. Munk, "Frequency Selective Surfaces: Theory and Design", Wiley, New York (2000) p.442.

[2] A.A.M. Saleh, R.A. Semplak, IEEE Trans., AP-24, (1976) 780.

[3] S. T. Chase, R.D. Joseph, Appl. Opt., 22, (1983) 1775.

[4] S. Gupta, G. Tuttle, M. Sigalas, K.-M. Ho, Appl. Phys. Lett., 71, (1997) 2412.

[5] D.R. Smith, J.B. Pendry, M.C.K. Wiltshire, Science, 305, (2004) 788.
[6] S. Govindaswamy, J. East, F. Terry, E. Topsakal, J. L. Volakis, G. I. Haddad, Microwave and Opt. Technol. Lett. , 41, (2004) 266.

[7] M.-H. Wu, K.E. Paul, J. Yang, G.M. Whitesides, Appl. Phys. Lett., 80, (2002) 3500.

[8] A.V. Kabashin, M. Meunier, C. Kingston, J.H.T. Luong, J. Phys. Chem. B, 107 (2003), 4527.

[9] E. Hutter, J.H. Fendler, Adv. Materials, 16, (2004) 1685.

[10]A. Taflove, S.C. Hagness, "Computational Electrodynamics: The Finite-Difference Time-Domain Method”, Artech House, Norwood, MA, (2000) p. 1038

[11]T. Kondo, S. Juodkazis, V. Mizeikis, S. Matsuo, H. Misawa, New J. Phys., 8, (2006) 250.

[12] P. Johnson, R. Christy, Phys. Rev. B, 6, (1972) 4730.

[13] K. Ueno, S. Juodkazis, V. Mizeikis, K. Sasaki, H. Misawa, J. Am. Chem. Soc., 128, (2006), 14226.

[14] K. Ueno, S. Juodkazis, V. Mizeikis, K. Sasaki, H. Misawa, Adv. Mater., 20, (2008) 26.

[15] K. Ueno, S. Juodkazis, T. Shibuya, Y. Yokota, V. Mizeikis, K. Sasaki, H. Misawa, J. Am. Chem. Soc., 133, (2008) 6928.

[16] K. L. van der Molen, K. J. Klein Koerkamp, S. Enoch, F. B. Segerink, N. F. van Hulst, L. Kuipers, Phys. Rev. B, 72, (2005) 45421.

[17] A. Degiron and T. W. Ebbesen, J. Opt. A: Pure Appl. Opt., 7, (2005) S90.

[18] J. H. Kim, P. J. Moyer, Opt. Express, 14, (2006) 6595.

[19] Z. Ruan, M.Qiu, Phys. Rev. Lett., 96, (2006) 233901.

(Received: July 10, 2009, Accepted: March 3, 2010) 